\title{
Endothelial Notch signaling is upregulated in human brain arteriovenous malformations and a mouse model of the disease
}

\author{
Patrick A Murphy', Gloria Lu', Steven Shiah", Andrew W Bollen² and Rong A Wang ${ }^{1}$
}

\begin{abstract}
Brain arteriovenous malformations (BAVMs) can cause lethal hemorrhagic stroke and have no effective treatment. The cellular and molecular basis for this disease is largely unknown. We have previously shown that expression of constitutively-active Notch4 receptor in the endothelium elicits and maintains the hallmarks of BAVM in mice, thus establishing a mouse model of the disease. Our work suggested that Notch pathway could be a critical molecular mediator of BAVM pathogenesis. Here, we investigated the hypothesis that upregulated Notch activation contributes to the pathogenesis of human BAVM. We examined the expression of the canonical Notch downstream target Hes1 in the endothelium of human BAVMs by immunofluorescence, and showed increased levels relative to either autopsy or surgical biopsy controls. We then analyzed receptor activity using an antibody to the activated form of the Notch1 receptor, and found increased levels of activity. These findings suggest that Notch activation may promote the development and even maintenance of BAVM. We also detected increases in Hes1 and activated Notch1 expression in our mouse model of BAVM induced by constitutively active Notch4, demonstrating molecular similarity between the mouse model and the human disease. Our work suggests that activation of Notch signaling is an important molecular candidate in BAVM pathogenesis and further validates that our animal model provides a platform to study the progression as well as the regression of the disease.
\end{abstract}

Laboratory Investigation (2009) 89, 971-982; doi:10.1038/labinvest.2009.62; published online 22 June 2009

KEYWORDS: angiogenesis; arteriovenous malformation; brain; endothelial cell; Notch signaling; stroke

Brain arteriovenous malformations (BAVMs) are characterized by a nidus of coiled, tortuous, and enlarged vascular lesions that shunt blood directly from feeding arteries to veins. ${ }^{1}$ They often rupture, resulting in hemorrhagic stroke in young people, most commonly between 20 and 40 years of age. ${ }^{1}$ BAVMs contribute to half of the hemorrhagic strokes in children, ${ }^{2}$ and $2 \%$ of all strokes. ${ }^{1}$ Currently, surgical resection is the primary treatment, but not all BAVMs can be removed safely. ${ }^{3}$ Most BAVMs are sporadic, making it difficult to identify the molecular cause by genetic association. ${ }^{1}$ To date, the cellular and molecular basis for BAVM pathogenesis remains largely unknown. This limited knowledge of BAVM etiology has impeded the rational design of molecular interventions.

Fundamentally, AVMs are a disruption of normal arteriovenous (AV) hierarchy, which was historically thought to be governed by hemodynamic forces. ${ }^{4}$ The discovery of genes with arterial or venous-specific expression in the developing mouse embryo has catalyzed advances in our understanding of the genetic control of $\mathrm{AV}$ specification and the establishment of AV hierarchy. ${ }^{5}$ Notch, a transmembrane receptor first identified in fruit fly, and involved in cell fate determination and tissue patterning throughout metazoans, has emerged as a critical mediator of AV differentiation. ${ }^{6}$ Studies in zebrafish and mouse development demonstrated that Notch signaling was necessary and sufficient for the expression of arterialspecific genes. ${ }^{6,7}$ Furthermore, we have demonstrated that endothelial Notch signaling regulates the luminal size of developing mouse arteries by promoting of arterial specification, and increasing the arterial allocation of endothelial cells. ${ }^{8}$ Abnormal Notch signaling induced

\footnotetext{
${ }^{1}$ Pacific Vascular Research Laboratory, Division of Vascular Surgery, Department of Surgery and ${ }^{2}$ Department of Pathology, University of California, San Francisco, CA, USA

Correspondence: Dr Rong A Wang, PhD., Department of Surgery, University of California, HSW 1618, Box 0507, 513 Parnassus Avenue, San Francisco, CA 94143-0507, USA.

E-mail: rong.wang@ucsfmedctr.org

Received 24 March 2009; revised 20 May 2009; accepted 21 May 2009
} 
enlarged AV connections and shunting in both mouse and zebrafish embryo, suggesting a link between AV specification and arteriovenous malformations (AVMs). ${ }^{6,7}$

Among the four mammalian Notch receptors and five ligands, Notch receptors 1 and 4 and their ligands Dll1, Dll4, and Jag1 are preferentially expressed in the arterial and not venous endothelium. ${ }^{9}$ Cell-cell mediated activation of the Notch receptor, by ligand binding to the extracellular domain, results in sequential cleavage events and release of an active intracellular domain (ICD).$^{10}$ Once cleaved, the ICD translocates to the nucleus, where it must form a complex with the sequence-specific DNA-binding protein Rbpj to promote the transcription of downstream genes. ${ }^{10}$ Transcription factors of the Hairy/Enhancer of Split (Hes) and Hes-related families of proteins, such as Hes1, are canonical target genes, and mediate many of Notch's downstream functions. ${ }^{10}$ Therefore, Notch-ICD is a constitutively active mutant. Likewise, the Notch4 mutant that lacks the extracellular domain is constitutively cleaved and activated $\left(\operatorname{Notch} 4^{*}\right) .{ }^{11}$ Thus, nuclear localization of cleaved NotchICD and expression of Hes1 are features of active Notch signaling.

To investigate whether upregulation of endothelial Notch signaling can disrupt AV hierarchy and cause AVMs in adult mice, we used a tetracycline-regulated transgenic system to express Notch $4^{\star}$ transgene specifically in the endothelium of adult mice (Notch4 $\left.{ }^{*}-\mathrm{Tet}\right)$, and reported AVMs in liver, skin, and uterus. ${ }^{12}$ Expression of the transgene in immature Notch $4^{*}$-Tet mice during post-natal brain growth resulted in hallmarks of BAVM in all mice, including enlarged and tortuous AV connections, shunting, and hemorrhagic stroke. ${ }^{13}$ In both adult and immature Notch4 ${ }^{*}$-Tet mice, the disease progression was reversed when the Notch $4^{*}$ transgene was turned off, demonstrating that Notch $4^{*}$ is critical to sustain the disease $\mathrm{s}^{12,13}$ The urgent question that arose out of this fundamental research is whether increased Notch signaling underlies the development and maintenance of human BAVM.

Notch loss-of-function mutations in $\mathrm{JAG1}, \mathrm{NOTCH} 3$, and NOTCH1 are known to cause Alagille syndrome, ${ }^{14}$ cerebral autosomal dominant arteriopathy with subcortical infarcts and leukoencephalopathy, ${ }^{14}$ and aortic valve anomalies, ${ }^{15}$ respectively, but it is not clear whether Notch signaling is involved in human BAVM pathogenesis. In this study, we test the hypothesis that Notch signaling is upregulated in human BAVMs by examining Notch-signaling activity in the endothelium of human BAVM relative to autopsy and surgical biopsy controls. We demonstrate increased levels of the activated-Notch1 receptor and canonical Notch target Hes1 in BAVM tissue. We reveal similar increases in our Notch4*-Tet mouse model of BAVM-like abnormalities. Our work puts forward the hypothesis that Notch activation causes and maintains human BAVMs, and provides molecular validation of our Notch4 $4^{*}$ Tet model of BAVM as a valuable system to dissect the molecular and cellular basis of BAVM pathogenesis.

\section{MATERIALS AND METHODS Clinical Samples}

The UCSF Committee on Human Research approved the use of human tissue samples for this study. BAVM samples and surgical biopsy controls were obtained by surgical resection and prepared by the UCSF hospital pathology lab. Samples were fixed in $10 \%$ neutral-buffered formalin, paraffin embedded, and cut at $5 \mu \mathrm{m}$. Control sections were either cerebral cortex or cerebellum. The cerebral cortex control sections were histologically normal temporal lobe from seizure resection cases or cerebral cortex from autopsy brains, which were histologically normal in patients without evidence of neoplastic disease. Cerebellar control sections were also from autopsy brains in patients with no evidence of neoplastic disease. In addition to two autopsy controls from UCSF, five autopsy controls were received as formalin-fixed sections from the Harvard Brain Tissue Resource Center, which is supported in part by PHS Grant number R24-MH 068855. These autopsy samples were also paraffin embedded and cut at $5 \mu \mathrm{m}$. Human small intestine biopsy was formalinfixed, paraffin-imbedded, and sectioned at $5 \mu \mathrm{m}$ by the UCSF hospital pathology lab. Snap frozen human small intestine tissue samples were provided by the Cooperative Human Tissue Network, which is funded by the National Cancer Institute, and sectioned at $10 \mu \mathrm{m}$.

\section{Mice}

Brain tissue was harvested from Notch4 ${ }^{*}$-Tet (Tie2-tTA;TRENotch $4^{*}$ ) mutants and littermate genetic controls (Tie2-tTA) at post-natal day $20 .{ }^{13}$ To suppress the gene expression, Tet sucrose solution $(0.5 \mathrm{mg} / \mathrm{ml}$ Tet, $50 \mathrm{mg} / \mathrm{ml}$ sucrose, Sigma $)$ was administered to pregnant mothers from plugging, and withdrawn from pups at birth as we described. ${ }^{13}$ All animals were treated in accordance with the guidelines of the UCSF Institutional Animal Care and Use Committee.

\section{Preparation of Mouse Tissue}

Endovascular labeling of perfused vessels was performed with FITC-lectin (Vector Labs, Burlingame, CA, USA) as described. ${ }^{13}$ Following perfusion with $1 \%$ paraformaldehyde (PFA) at $100 \mathrm{~mm} \mathrm{Hg}$, brain tissue was fixed overnight in $4 \%$ PFA, and then dehydrated in $70 \%$ ethanol in water, and $100 \%$ ethanol before xylene treatment and paraffin embedding. Small and large intestine was fixed overnight in 4\% PFA, and paraffin-embedded according to the methods used for brain tissue.

\section{Immunofluorescent Staining}

For the purposes of comparison, BAVM sections were always stained with control sections. Tissue sections were deparaffinized in xylene and rehydrated. Following antigen retrieval by sodium citrate, the samples were blocked with Avidin/Biotin Blocking Kit (Vector Labs) and then 10\% goatserum and $0.2 \%$ Triton X-100 in PBS. Hes1-stained samples were also treated with $500 \mathrm{U} / \mathrm{ml}$ DNAse I for $10 \mathrm{~min}$ at $37^{\circ} \mathrm{C}$ 
(Promega, Madison, WI, USA) before blocking. Primary treatment was performed overnight at $4{ }^{\circ} \mathrm{C}$ in block. Secondary treatment was performed with biotinylated antirabbit or anti-mouse antibodies (Vector Labs) in block. Tertiary treatment was performed with streptavidin-Cy3 in PBS (JacksonImmuno, West Grove, PA, USA). Slides were stored in VectaShield plus DAPI (Vector Labs).

\section{Antibodies}

Hes1 antibody was kindly provided by Dr Nadean Brown (at the Children's Hospital Medical Center, Cincinnati, OH, USA). We also used activated-Notch1 antibody (Val1744, Cell Signaling, Beverly, MA, USA) and human CD31 antibody (JC70/A, DAKO, Carpinteria, CA, USA).

\section{Data Analysis}

Stained tissue sections were imaged using a $\times 40$ objective on a Zeiss Axiovert fluorescent microscope (Thornwood, NY, USA) with Intelligent Imaging software (Denver, CO, USA). In BAVM sections, the three or four vessels with the strongest endothelial cell staining were imaged. Large vessels with a thick media, characteristic of arteries, were imaged. In autopsy control and surgical biopsy control samples, three or four vessels of similar caliber to the BAVM vessels were imaged. CD31 or non-specific IgG was imaged in the same vessel in adjacent sections. The same exposure time was used for all slides stained with a given antibody and the nonspecific IgG control. Individual fluorescent channel intensities were exported as 16-bit TIFF files and analyzed by a blinded examiner using ImageJ. The examiner picked the three cells, lining each vessel lumen, which showed the most intense Hes1 or activated-Notch1 staining by eye. They then circumscribed the DAPI-labeled nuclei of these cells, and measured the mean intensity of Hes1 or activated-Notch1 signal in the enclosed area. Paraffin sections of brain from Notch4 ${ }^{*}$-Tet (Tie2-tTA:TRE-Notch4 ${ }^{*}$ ) mutants and genetic controls (Tie2-tTA) were processed in the same way. Hes1 and activated-Notch1 staining in the nuclei of endothelial cells of mouse tissue was normalized to non-specific IgG staining. Staining in human tissue is shown as unadjusted mean intensity values.

\section{Statistical Analysis}

Each individual case or control, consisting of at least three intensity measurements from each of three separate vessels, was processed to provide a mean value and s.e.m. A two-tailed Wilcoxon's rank sum non-parametric test was performed with STATA-IC (College Station, TX, USA) to determine the significance of the difference between these mean values from the BAVM cases relative to those of the autopsy controls. The same analysis was performed to determine the significance of the difference in mean values between BAVM cases and surgical biopsy controls.

\section{Confocal Imaging of Crypt Cells}

For nuclear localization of Hes1 staining in the crypt cells of human small intestine, sections were imaged using a $\times 63$ oil-immersion objective on a Zeiss LSM510 microscope. The same exposure settings were used for Hes1-stained samples and IgG control.

\section{RESULTS \\ Notch Signaling Pathway Activity is Increased in the Endothelium of Human BAVMs}

To examine Notch activity in BAVMs we analyzed the expression of the canonical Notch downstream target Hes1 in endothelium of human BAVMs by immunofluorescence. We chose Hes1 because it is a direct transcriptional target of activated Notch both in vitro ${ }^{16,17}$ and in vivo ${ }^{18,19}$ and because Hes1 antibodies have been wellcharacterized in immunostaining of Notch gain- and lossof-function tissue. ${ }^{20-24}$ To verify the specificity of the Hes 1 antibody in our brain samples, we examined Hes1 staining in a control. In the mouse intestine, Hes1 expression has been well characterized in the nuclei of the crypt cells at the base of the villi. ${ }^{22,24-26}$ We found that the Hes1 antibody staining faithfully replicated the established pattern in both paraffinfixed (Figure 1a, b) and fresh frozen (data not shown) mouse large and small intestine. To confirm the specificity of the Hes1 antibody in human tissue, we also stained paraffin-fixed (Figure 1e) and fresh frozen (data not shown) human small intestine, and found that the Hes1 antibody stained the nuclei of crypt cells in the human tissue as well (Figure 1e-3). As a negative control, we performed staining of adjacent sections of small intestine with the same concentration of non-specific IgG, and did not observe similar patterns (Figure 1c, d, f).

We then stained paraffin sections of human BAVMs by immunofluorescence (Supplementary Table 1). We detected Hes1 protein in areas of the endothelium of human BAVMs (Figure 2b), where it was found in the nuclei of endothelial cells (ECs), consistent with the nuclear localization of the transcription factor (Figure 2b-1).

To determine whether Notch activity was increased in the endothelium of human BAVMs, we compared staining intensity with control human brain sections from autopsy (Supplementary Table 1). We detected little or no Hes1 in autopsy controls, although strong CD31 staining confirmed the endothelium was intact in these samples (data not shown). We quantified this difference by measuring the fluorescent intensity of nuclear Hes1 in the most strongly stained EC nuclei in BAVM samples, and comparing this to controls. We found that the average Hes1 intensity in the strongly stained areas of BAVMs is significantly higher than autopsy controls (Figure 2f; $N=12$ BAVM samples, $N=7$ controls; $P=0.001$ ). Among individual samples, 9 of 12 BAVM samples had higher mean Hes1 intensity than the most intense autopsy control. As 


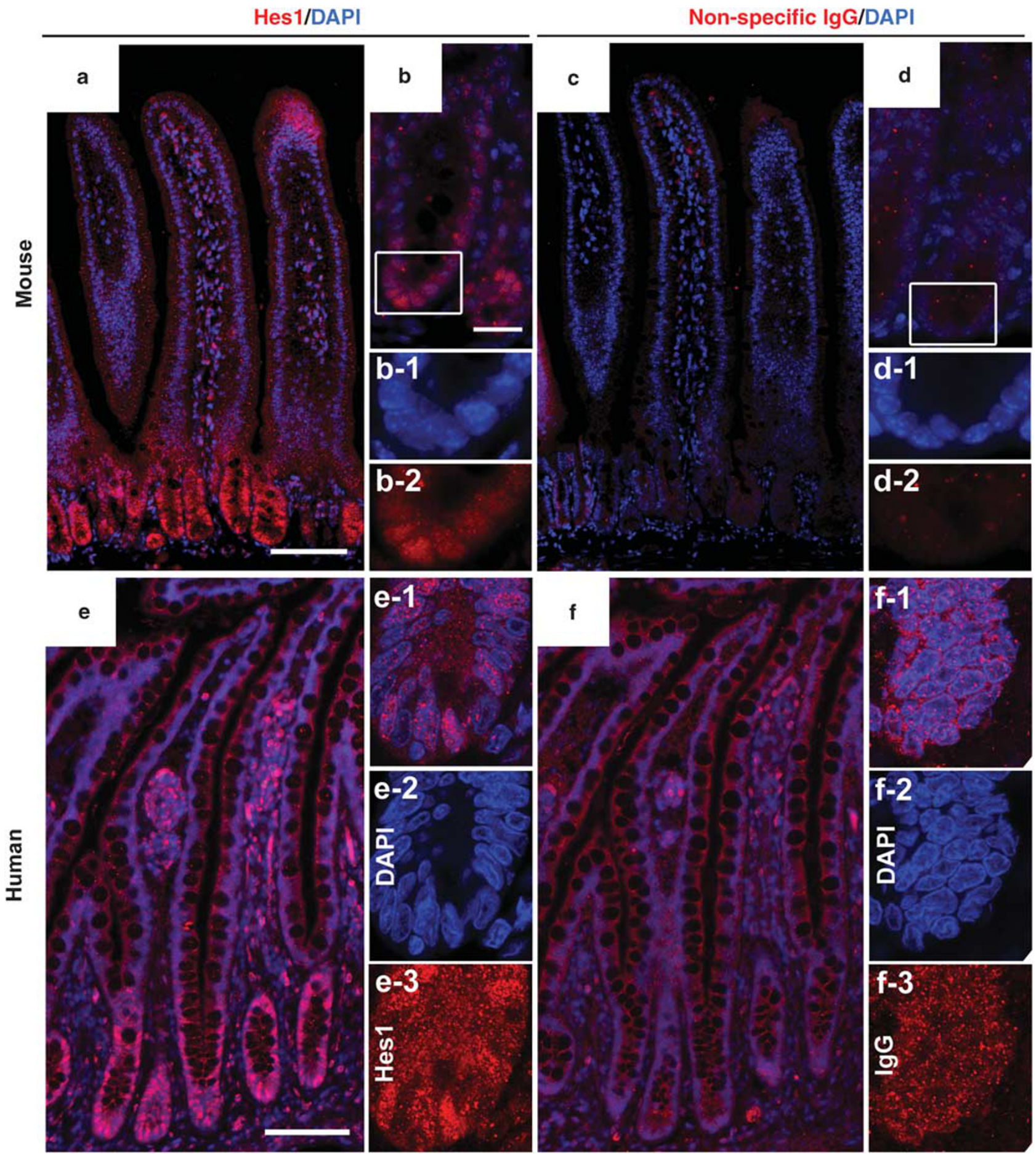

Figure 1 Hes 1 staining of crypt cell nuclei of the gut confirms antibody specificity. (a, b) Positive control stained with Hes1 antibody. Typical staining pattern shown by immunofluorescence in the crypt cells of mouse large (a) and small intestine (b), demonstrates specificity of the Hes1 antibody. High magnification of the crypt (boxed area in b) shows co-localization of Hes1 and DAPI nuclear label (b-1 to b-2). (c-d) Negative control for Hes1 staining, nonspecific IgG in adjacent sections. (e) Typical staining pattern shown by immunofluorescence in the crypt cells of human small intestine, demonstrates the specificity of the Hes1 antibody. High magnification confocal imaging of the crypt shows co-localization of Hes1 and DAPI nuclear label (e-1 to e-3). (f) Negative control with non-specific lgG in an adjacent section to (e) does not show the same staining pattern. Scale bars (a \& c) $100 \mu \mathrm{m},(\mathbf{b} \& \mathbf{d}) 50 \mu \mathrm{m},(\mathbf{e} \& \mathbf{f})$ $100 \mu \mathrm{m}$.

a negative control, we used a non-specific IgG primary antibody on adjacent tissue sections, and did not see a similar staining pattern (Figure 2c).
Autopsy samples are excellent controls for human BAVM because they are screened for the absence of any detectable brain pathologies, however they are not subject to the same 

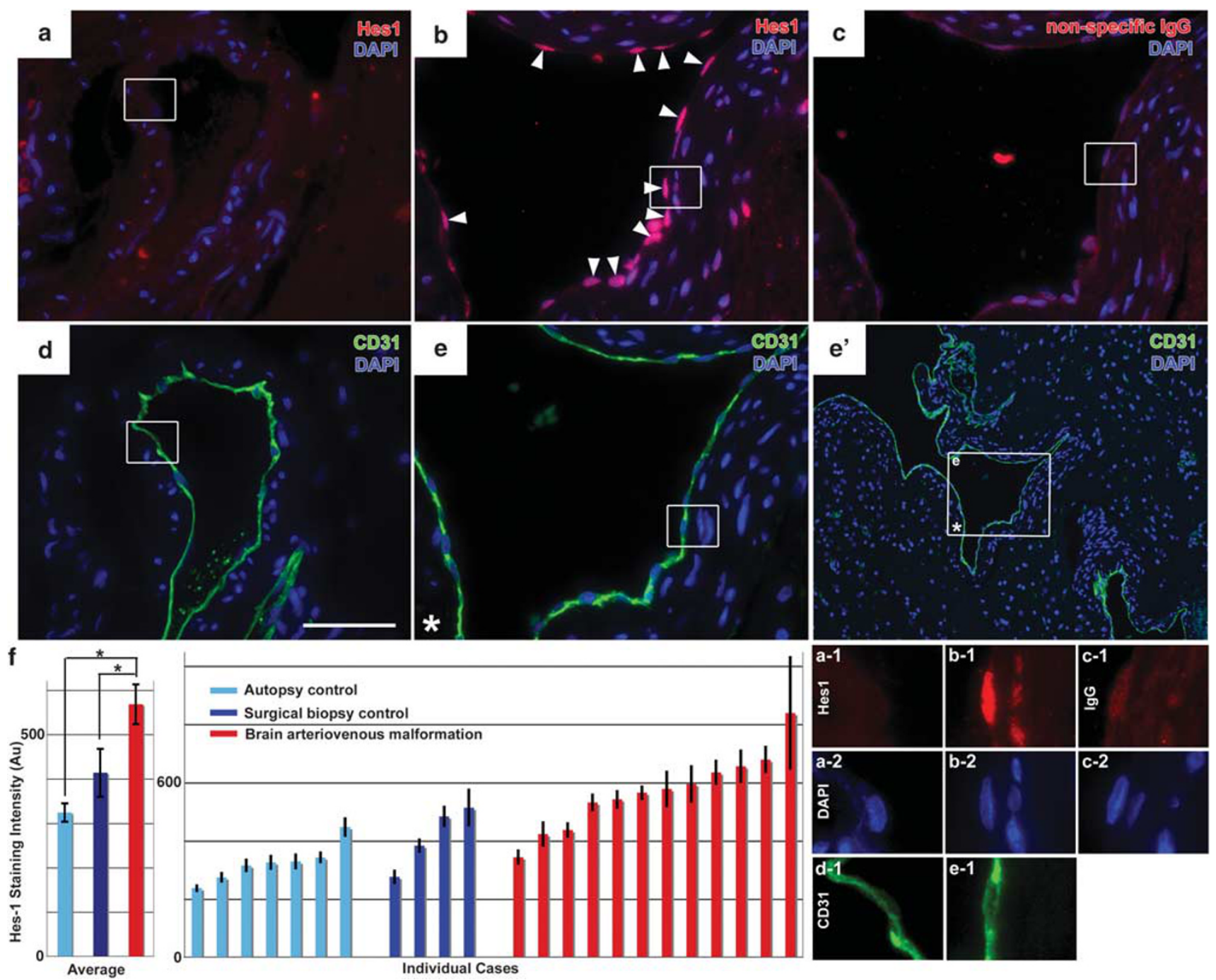

Figure 2 Increased Hes1 staining in the endothelium of human brain arteriovenous malformations (BAVMs). (a, b) Immunofluorescent staining for Hes1 in paraffin sections from surgical biopsy controls (a) and human BAVMs (b). Increased Hes1 staining is evident in the endothelium of human BAVMs (arrowheads, b). (c) Negative control for Hes1 staining, non-specific IgG in an adjacent section to (b). (d \& e) CD31-stained sections adjacent to (a \& b), respectively. Low-magnification (e') shows the location of (e) within a large vascular structure (box with asterisk). High magnification of the boxed areas in (a-e) shows co-localization of Hes1 staining with the DAPI nuclear label (a-1 to c-1 and a-2 to c-2) in cells lining CD31-labeled vessels (d-1 \& e-1).

(f) Quantification of Hes1 staining in BAVM cases and autopsy or surgical biopsy controls. Scale bars (a-e) $200 \mu \mathrm{m}$. In graph ( $N=12$ BAVM samples, $N=7$ autopsy controls, $N=4$ biopsy controls; BAVM vs autopsy controls $P=0.0003$; BAVM vs biopsy controls $P=0.05$ ). Average represents mean \pm s.e.m. of cases. Individual cases represent mean \pm s.e.m. of individual endothelial cells in each case.

surgical manipulation as BAVM biopsies, and not fixed as quickly. This difference in tissue handling is particularly important because Hes1 can be degraded within hours in some non-endothelial cell types. ${ }^{27}$ Therefore, we also examined levels of Hes1 staining in ECs of surgical brain biopsies from patients without BAVM (Supplementary Table 1), controls which are subject to the same manipulation and fixation as BAVM samples. Since brain biopsies are not taken from patients with normal brain function, we have selected biopsy samples from the least affected patients, to minimize the potential effects of brain pathology. The combination of these controls provides the most vigorous baseline expression. We found that, as in the autopsy controls, levels of Hes1 staining in the nuclei of ECs was low or absent (Figure 2a, a-1). We quantified staining intensity in these samples, as we did in the autopsy controls. We found that average Hes1 intensity in BAVMs is significantly higher than in biopsy controls (Figure 2f; $N=12$ BAVM samples, $N=4$ controls; $P=0.039$ ). Among individual samples, 9 of 12 BAVM samples had higher mean Hes1 intensity than the most intense surgical biopsy control. As a negative control, we used a non-specific IgG primary antibody on adjacent tissue sections, and did not see a similar staining pattern (Figure 2c). CD31 staining confirmed the integrity of the endothelium in tissue sections from BAVMs (Figure 2e) and surgical biopsy controls (Figure 2d). 

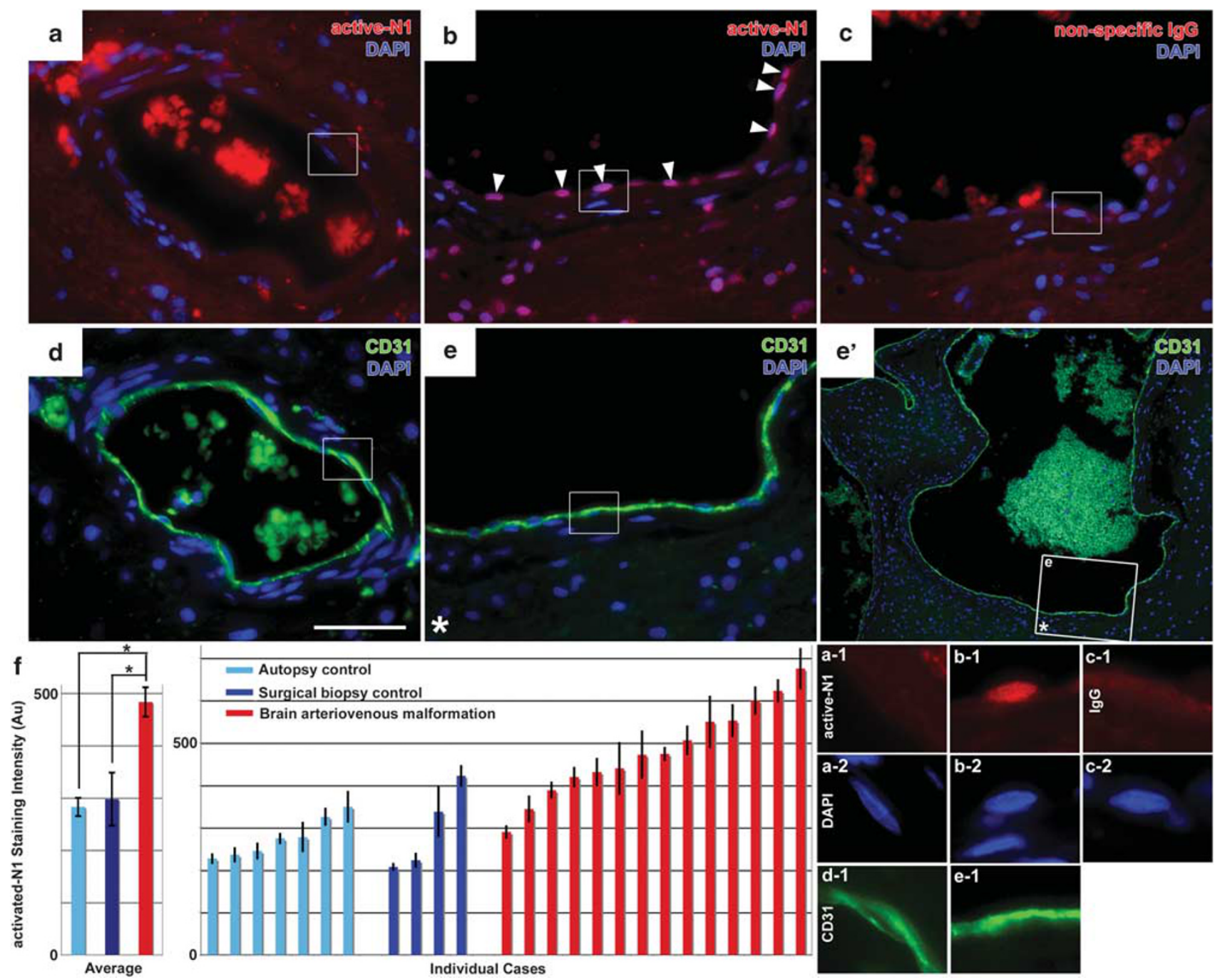

*

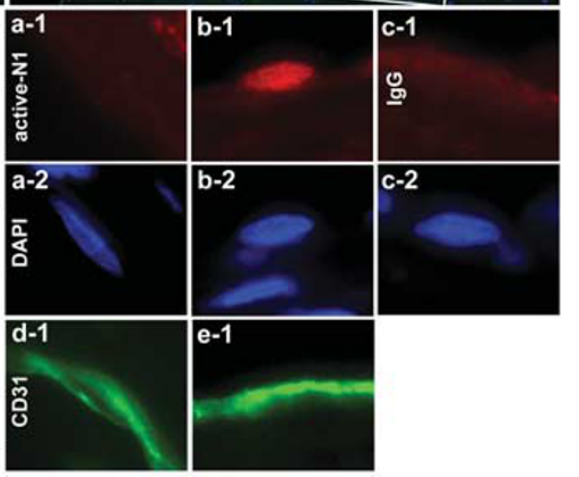

Figure 3 Increased activated-Notch1 staining in the endothelium in human brain arteriovenous malformations (BAVMs). (a, b) Immunofluorescent staining for activated-Notch1 in paraffin sections from surgical biopsy controls (a) and human BAVMs (b). Increased activated-Notch1 staining is evident in the endothelium of human BAVMs (arrowheads, b). (c) Negative control for activated-Notch1 antibody staining, non-specific lgG in an adjacent section to (b). (d \& e) CD31 stained adjacent sections to $(\mathbf{a} \& \mathbf{b})$ respectively. Low-magnification (e') shows the location of the stained area within a large vascular structure (box with asterisk). (f) High magnification of the boxed areas shows co-localization of activated-Notch1 staining with the DAPI nuclear label (a-1 to c-1 and a-2 to c-2) in cells lining the CD31-labeled vessels (d-1 \& e-1). (f) Quantification of activated-Notch1 staining in BAVM cases and autopsy or surgical biopsy controls ( $N=14$ BAVM samples, $N=7$ autopsy controls, $N=4$ biopsy controls; BAVM vs autopsy controls $P=0.0001$; BAVM vs biopsy controls $P=0.008$ ). Scale bar is $200 \mu \mathrm{m}$. Average represents mean \pm s.e.m. of cases. Individual cases represent mean \pm s.e.m. of individual endothelial cells in each case.

To examine Notch receptor activation directly, we measured the levels of activated-Notch1 by immunofluorescence in our sample set. We chose to use the activatedNotch1 antibody because it has previously been validated in mouse tissue ${ }^{28,29}$ and also detects human forms of activatedNotch $1,{ }^{30}$ since it was raised against a human antigen. As with Hes1, we found that ECs in some of the vessels of BAVM biopsy were strongly positive (Figure $3 \mathrm{~b}$ ). In these ECs, we detected activated-Notch1 in the nucleus, consistent with the nuclear translocation of the activated receptor (Figure 3b-1). As a negative control, we stained adjacent sections of brain with the same concentration of non-specific IgG, and did not observe the same pattern (Figure 3c, c-1). Nuclear staining in autopsy or surgical biopsy controls was not as intense as in BAVM samples (Figure 3a, a-1). We quantified differences in activated-Notch1 staining between BAVM samples and autopsy or surgical biopsy controls, and found that average activated-Notch1 intensity in BAVM is significantly higher than autopsy controls (Figure 3f; $N=14$ BAVM samples, $N=7$ autopsy controls; $P=0.0006)$, and surgical biopsy controls (Figure 3f; $N=4$ biopsy controls; $P=0.015$ ). Among individual samples, 12 of 14 BAVM samples had higher mean activated-Notch1 intensity than the most intense autopsy control, and 10 of 14 had higher mean activated-Notch1 intensity than the most intense surgical biopsy control. 


\section{Notch-signaling Pathway Activity is Increased in Murine BAVM-like Abnormalities Caused by Endothelial-specific Expression of Notch4* \\ We previously reported that endothelial expression of} Notch4 ${ }^{*}$, encoding the truncated intracellular domain of Notch4, results in Notch4* activity, indicated by nuclear localization of the intracellular domain of the Notch4 receptor. ${ }^{13}$ However, because Notch4 antibody staining cannot differentiate between the Notch $4^{*}$ transgene and endogenous Notch4, we were unable to determine whether Notch $4^{\star}$ expression increased endogenous Notch signaling. We also demonstrated the increased expression of Notch downstream genes in whole tissue homogenate from Notch $4^{*}$-Tet mice, ${ }^{12}$ but the lack of spatial resolution prevented the identification of the cells in which Notch signaling was activated. Here, we tested whether endogenous Notch1 activity and expression of Notch downstream target Hes1 is upregulated in the endothelium of Notch4 ${ }^{\star}$-Tet mice.

To determine whether expression of Notch $4^{*}$ increased Notch-signaling activity in the endothelium during BAVM formation in Notch4 ${ }^{*}$-Tet mice, we examined the expression of the canonical Notch downstream gene Hes1 in paraffinfixed brain sections from Notch4*-Tet mice with BAVM-like abnormalities. We detected Hes1 in the nuclei of ECs, consistent with the nuclear localization of the transcription factor (Figure $4 \mathrm{a}, \mathrm{a}-1$ ). To control for non-specific staining, we stained an adjacent section with non-specific IgG of the host species used to generate the Hes1 antibody, at the same concentration as the specific primary (Figure $4 c, c-1$ ). Nuclear staining in genetic controls was not as intense as in Notch $4^{*}$-Tet mice (Figure 4b, b-1). Antibody staining intensity was calculated from the ratio of Hes1 staining to nonspecific IgG staining. We found an increase in Hes1 signal intensity in the Notch $4^{\star}$-expressing mice, relative to controls (Figure 4d; $N=5$ controls, $N=3$ Notch $4^{*}$ Tet mutants; $P=0.0253)$

To determine whether expression of Notch $4^{*}$ increased levels of endogenous Notch signaling, we examined the levels of activated-Notch1 in the endothelium in paraffin-fixed brain sections from Notch4 ${ }^{\star}$-Tet mice using an antibody to the activated form of Notch1. We detected activated-Notch1 in the nuclei of ECs, consistent with the nuclear translocation of the activated-Notch1 receptor (Figure 5a, a-1). We controlled for non-specific staining and quantified staining intensity as we had for Hes1 (Figure 5c). Nuclear staining in genetic controls was not as intense as in Notch4*-Tet mice (Figure 5b, b-1). We found increased levels of activatedNotch1 signal intensity in Notch $4^{*}$-Tet mice, relative to controls (Figure 5d; $N=5$ controls, $N=3$ Notch $^{*}$-Tet mutants; $P=0.0253$ ).

In summary, we show that Notch signaling is increased in human BAVM, using the same tissue preparation, antibodies, and quantification that we use to show increased Notchsignaling in our Notch4 ${ }^{\star}$-Tet transgenic mouse model of the disease.

\section{DISCUSSION}

Our study demonstrates that Notch activity is increased in human BAVMs, supporting the hypothesis that Notch activation causes and maintains human BAVM.

We found increased levels of the canonical Notch downstream gene Hes1 in the endothelium of human BAVMs relative to levels in autopsy or surgical biopsy controls, as well as increased levels of activated-Notch1 receptor. Furthermore, we demonstrate molecular similarity between the BAVM-like abnormalities of our Notch4 ${ }^{*}$-Tet mouse model and human BAVM, providing molecular validation for this model of the human disease.

\section{Notch Signaling is Increased in the Endothelium of Human BAVM}

Hes1 is a canonical Notch downstream gene, and increased endothelial Hes1 expression indicates increased endothelial Notch pathway activity. Hesl is a direct transcriptional target of Notch activity. ${ }^{16}$ In the vascular endothelium, Hes 1 expression is regulated by Notch activity. Transfection of human umbilical vein endothelial cells with Notch4* resulted in an approximately sixfold increase in Hesl expression by quantitative PCR. ${ }^{17}$ Hes1 expression is also increased by the induction of endogenous endothelial Notch signaling in vitro ${ }^{31}$ and general Notch signaling in vivo. ${ }^{32}$ Conversely, interference with endogenous endothelial Notch signaling decreases Hesl expression in vitro. ${ }^{31}$ and in vivo ${ }^{18,19}$ Therefore, increased Hes1 expression in the endothelium of human BAVMs indicates increased Notch-signaling activity.

The specificity of Hes1 staining in BAVM is supported by extensive evaluation in mouse and human tissue. We systematically tested five Hes1 antibodies on frozen and paraffin-fixed tissue, and found that only one gave the expected staining pattern in the positive controls, intestinal crypt cells in mouse and human tissue. We chose this positive control because the distinctive crypt-specific Hes1 expression pattern has been repeatedly demonstrated at both the protein and RNA level by several investigators. ${ }^{22,24-26}$ Demonstrating its dependence on Notch signaling, Hes1 protein expression in the crypt cells is lost when Notch receptors are deleted in the crypt cells, or the $\gamma$-secretase activity required for Notch receptor activation is pharmacologically blocked. ${ }^{22,24}$ The specificity of staining with this particular Hes1 antibody has been reported in both frozen and paraffin-fixed mouse tissue. $^{20,21,33}$ Although the mouse-derived antigen used to generate the antibody is $90 \%$ similar to human Hes $1,{ }^{20}$ confirmation of Hes1 staining in human crypt cells was a critical step to verify the antibody-detected Hes1 in human as well as mouse tissue. Our finding of increased Hes1 expression in the endothelium of human BAVMs is strongly supported by the validation of antibody specificity in positive controls.

Notch1 is a critically important receptor in the endothelium, and the upregulation of Notch1 activity that we 

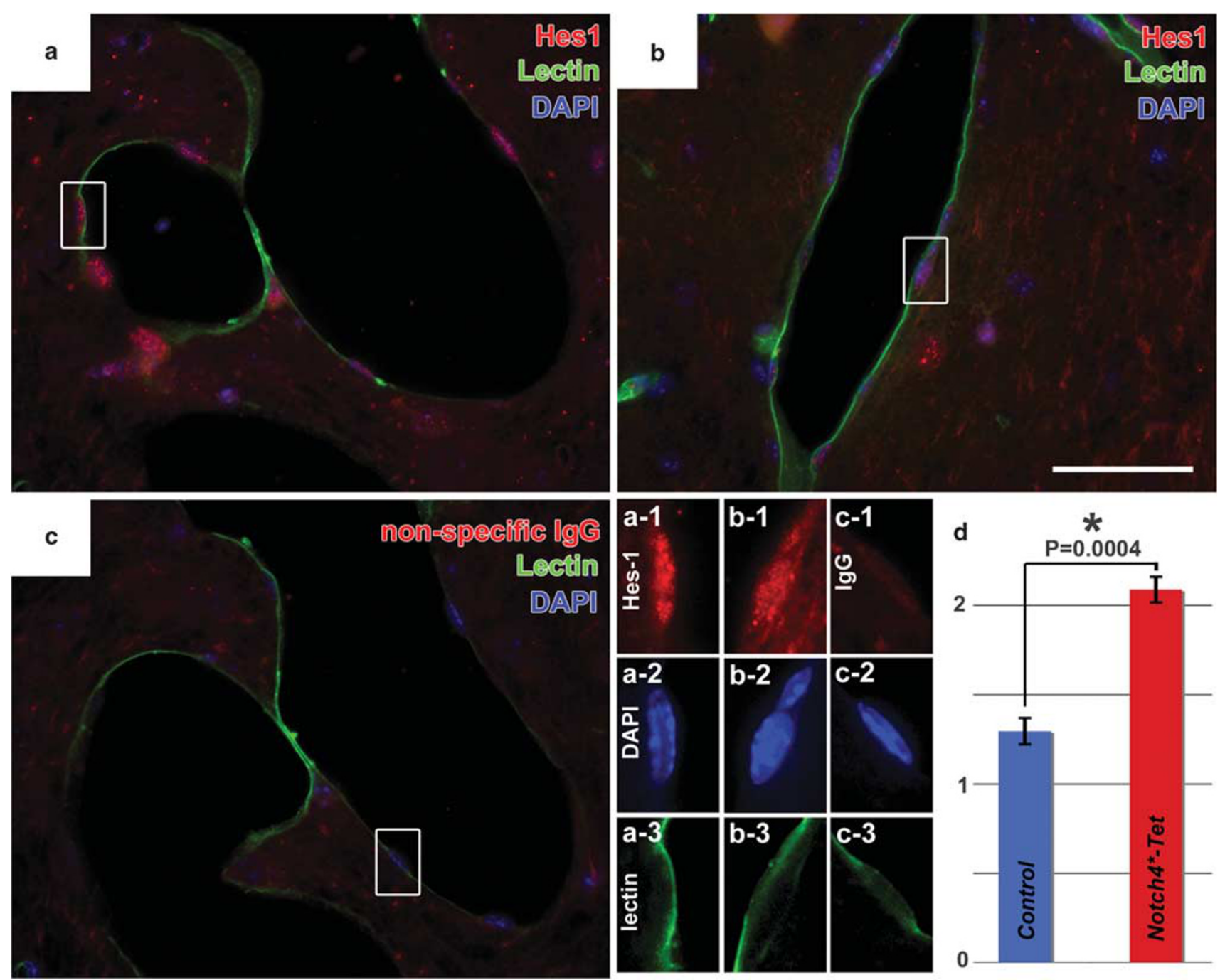

Figure 4 Increased Hes1 staining in the endothelium of the Notch4*-Tet mouse model of brain arteriovenous malformation (BAVM)-like lesions.

(a-b) Immunofluorescent staining for Hes1 in paraffin sections from Notch4*-Tet mice (a) and genetic controls (b). Increased Hes1 staining is evident in the endothelium of FITC-lectin perfused vessels in Notch4*-Tet mice. (c) Negative control for Hes1 staining, non-specific lgG in an adjacent section to (a). High magnification of the boxed areas shows co-localization of Hes 1 staining with the DAPI nuclear label (a-1 to c-1 and a-2 to c-2) in cells lining the lectinperfused vessel lumen (a-3 to c-3). (d) Quantification of Hes1 staining relative to non-specific lgG staining in Notch4*-expressing mutant mice and controls $\left(N=5\right.$ genetic controls, $N=3$ Notch $4^{*}$-Tet mutants, values represent mean \pm s.e.m., $\left.P=0.0004\right)$. Scale bars $(a-c) 200 \mu \mathrm{m}$.

have demonstrated in human BAVM may be involved in the development and progression of the disease. Notch1 is necessary for vascular development, and subtle changes in Notch1 levels in humans and mice can cause severe vascular defects. ${ }^{6}$ In humans, inheritance of a single mutated loss-offunction allele of Notch 1 can cause aortic valve disease and increase the risk of thoracic aorta anuerysm. ${ }^{15,34}$ Studies in mice demonstrated the importance of endothelial Notch1 signaling, because endothelial-specific deletion of just one Notch1 allele significantly impaired blood flow recovery in the hindlimb after the femoral artery was occluded, ${ }^{31}$ and deletion of both alleles is embryonic lethal. ${ }^{8,31}$ At a cellular level, Notch1 deletion in endothelial cells increases their contribution to angiogenic sprouts rather than retention in existing vessels. ${ }^{36}$ Expression of the constitutively active in- tracellular domain of the Notch1 receptor (Notch1-ICD) specifically in the endothelium is also embryonic lethal ${ }^{37}$ (our own unpublished data). We found that even at an adult stage, expression of Notch $1^{*}$ in the endothelium was sufficient to cause vascular malformations, ${ }^{12}$ demonstrating a requirement for Notch-signaling homeostasis in adult mice. Therefore, the requirement for tightly regulated Notch1 signaling in the endothelium suggests that the increased activity we observed may disrupt vascular organization.

The cause for upregulated Notch activity in the endothelial cells of BAVM is not yet apparent. Elevated activity may be a secondary effect of BAVM formation. For example, the endothelial cells of BAVMs are exposed to a massive increase in blood flow, which has been shown to increase the expression of Notch ligand Jag1, Notch4 receptor, and down- 

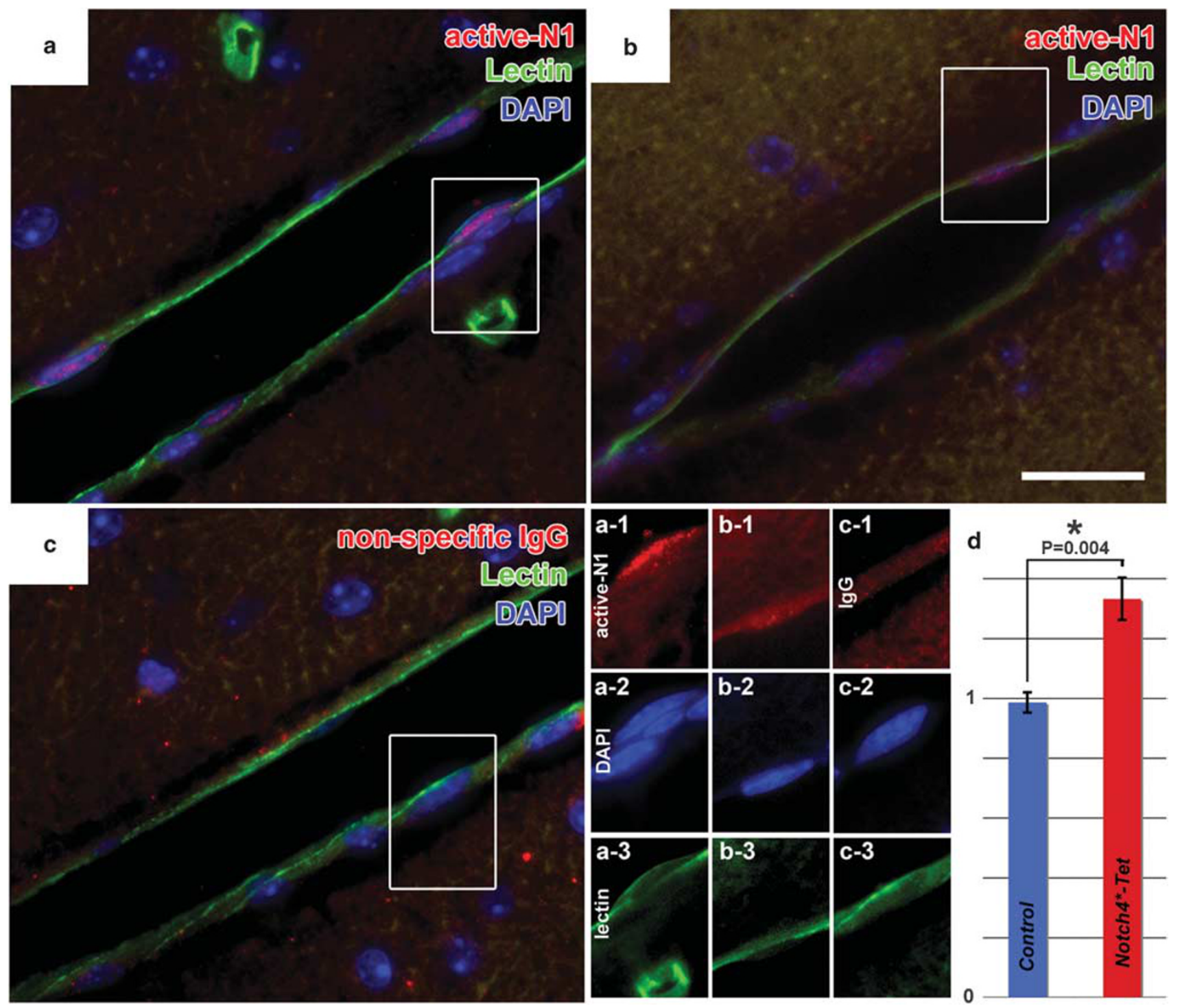

Figure 5 Increased activated-Notch1 staining in the endothelium of the Notch4*-Tet mouse model of brain arteriovenous malformation (BAVM)-like lesions. (a, b) Immunofluorescent staining for activated-Notch1 in paraffin sections from Notch4*-Tet mice (a) and genetic controls (b). Increased activated-Notch1 staining is evident in the endothelium of FITC-lectin perfused vessels in Notch4*-Tet mice (a). (c) Negative control for activated-Notch1 staining, non-specific IgG in an adjacent section to (a). High magnification of the boxed areas shows co-localization of activated-Notch1 staining with the DAPI nuclear label (a-1 to c-1 and a-2 to c-2) in cells lining the lectin-perfused vessel lumen (a-3 to c-3). (d) Quantification of activated-Notch1 staining relative to non-specific lgG staining in Notch $4^{*}$-expressing mutant mice and controls. $\left(N=5\right.$ genetic controls, $N=3$ Notch $4^{*}$-Tet mutants, values represent mean \pm s.e.m., $\left.P=0.004\right)$. Scale bar is $100 \mu \mathrm{m}$.

stream genes Heyl and ephrin-B2 in endothelial cells in vitro. ${ }^{38,39}$ However, our animal data have demonstrated that increased Notch4 signaling can initiate BAVM-like pathology in mice, ${ }^{13}$ suggesting that the elevated Notch activity may be a causal molecular lesion. Therefore, increased Notch activity seen in human BAVM may not only be a secondary effect of increased blood flow, but may also be an initial molecular pathology.

The increase in Notch activity in the endothelium of human BAVM appears subtle, but this small change is likely sufficient to cause biological effects. Haploinsufficiencies of Notch $1^{15,34}$ or its ligands Dll1, ${ }^{35}$ Dll4, ${ }^{18,40,41}$ and Jag1 ${ }^{42}$ impair vascular function in mice and humans. Of the ligands, Dll4 may be the most critical, because haploinsufficiency of this gene is embryonic lethal. ${ }^{18,40,41}$ Such sensitivity in the endothelium to receptor or ligand dosage is rare; vascular endothelial growth factor A (Vegfa) is the only other protein known to exert such profound vascular effects as a result of haploinsufficiency. ${ }^{43,44}$ Other biological systems have shown a similar threshold requirement for Notch activity. For example, in left-right differentiation in the chick, a transient increase in extracellular calcium slightly increases Notch receptor activation on the left side of the developing embryo, ${ }^{45}$ resulting in the subsequent establishment of asymmetry. 
Similarly, in neuronal development in the fly, small changes in Notch activity between daughter cells are necessary to establish asymmetric fates, and mutations that cause either a general increase or decrease in Notch signaling results in loss of asymmetry. ${ }^{46}$ Therefore, even small changes in the level of Notch activity have the potential to cause severe vascular effects.

\section{Notch Activation may be a Key Regulator of BAVM Development}

Our study demonstrates increased Notch-pathway activation in the endothelium of human BAVM. Until now, the involvement of Notch signaling in the development of human BAVM has not been reported. Genetic understanding of human BAVM has been limited by the sporadic nature of the disease. An exception is the development of BAVM in 10$20 \%$ of patients suffering from the autosomal-dominant disease Hereditary Hemorrhagic Telangectasia (HHT). ${ }^{47}$ HHT causes widespread AVMs through many tissues, including the brain, and has been linked to mutations in the TGF- $\beta$ receptors Alk1 (ACVRL1) and endoglin $(E N G) .{ }^{48} \mathrm{Al}-$ though HHT is implicated in only $2 \%$ of all BAVM, ${ }^{49}$ it has been the most studied pathway in human BAVM due to this genetic association. In mouse models, homozygous mutations in these receptors are embryonic lethal, and heterozygous mutations result in vessel enlargement and hemorrhage, although high-flow arteriovenous shunting characteristic of BAVMs has not been reported. ${ }^{50-55}$ The low penetrance and focal development of BAVMs in HHT suggest that other causes, potentially other signaling pathways, cooperate with the TGF- $\beta$ mutations to cause this pattern of BAVM development.

Other endothelial-signaling pathways appear to be upregulated in human BAVM samples, although animal studies have not demonstrated that these changes induce BAVM. VEGFA expression in the BAVM nidus has been observed at both the RNA and protein level and is increased at the RNA level in whole-tissue homogenate of the BAVM nidus relative to control brain biopsies. ${ }^{56}$ However, while forced expression of VEGFA in the mouse brain results in increased angiogenesis, development of AV shunting has not been reported. ${ }^{57-61}$ Similarly, increased expression of angiopoietin-2 (ANGPT2) has been reported at the RNA and protein level in the BAVM nidus ${ }^{56,62}$ but expression of ANGPT2 in the adult mouse brain has not been reported to result in AV shunting. ${ }^{59}$ Gain- and loss-of-function mutations in ANGPT2's endothelial receptor, TIE2 (TEK), have been associated with venous malformations, but not AVMs. ${ }^{63}$ Integrin alpha $V$ (ITGAV) was also upregulated in the endothelium and smooth muscle cells of BAVMs. ${ }^{56}$ However, no gain-offunction animal studies have been reported. Complete endothelial deletion of Itgav causes no detectable cerebrovascular defects, ${ }^{64}$ although deletion of Itgav in neuronal cells results in dilation of blood vessels and hemorrhage, ${ }^{64}$ suggesting a role in vascular stability through paracrine effects, but not cell autonomous vascular effects. Therefore, the molecular basis of BAVM pathogenesis remains largely unknown.

Increased endothelial Notch signaling is sufficient to induce vascular abnormalities with the hallmarks of BAVM in Notch $4^{*}$-Tet mice, and an increase in both Hes1 and activated-Notch1 protein in Notch $4^{*}$-Tet mice demonstrates similarity at the molecular level to the human disease. We have reported that expression of constitutively active Notch4 receptor in the endothelium of our Notch4 $4^{\star}$-Tet mice results in enlarged and tortuous BAVM-like vascular abnormalities, shunting of blood, and hemorrhagic stroke. ${ }^{13}$ Here, we demonstrate that Notch activity is increased in the endothelium of human BAVM, as it is in Notch4*-Tet mice. We found similar increases in Hes1 and activated-Notch1 in human BAVM and BAVM-like abnormalities in Notch4 ${ }^{*}$-Tet mice. One key difference between human BAVM and the mouse model is that human BAVMs are focal, ${ }^{1}$ whereas the BAVMlike abnormalities in Notch $4^{*}$-Tet mice are pervasive. ${ }^{13}$ This is likely a consequence of the transgenic expression of constitutively active Notch4 throughout the endothelium in the mouse model. Therefore, increased expression of Hes1 and activated-Notch1 demonstrates molecular similarity between BAVM-like abnormalities in Notch4 ${ }^{*}$-Tet mouse model, caused by increased endothelial Notch activity, and human BAVMs.

It is commonly thought that, once they develop, BAVMs do not regress, but remain as a constant threat of hemorrhagic stroke. ${ }^{65}$ Our animal studies have suggested the tantalizing possibility that, in animals at least, BAVM-like lesions are reversible. ${ }^{13}$ The dependence of BAVMs on the activity of molecular-signaling pathways is a novel concept, but we have also shown that AVMs that form in the livers of Notch4*-Tet mice regress completely after the Notch $4^{*}$ transgene is suppressed, demonstrating that endogenous machinery exists for the reversal of AVMs once the causative molecular lesion is removed. ${ }^{12}$ Our human data does not demonstrate that increased Notch activity promotes the development and growth of BAVM in humans. Nonetheless, we found that Notch activity is upregulated in a large portion of human BAVMs, after their initiation, suggesting that increased Notch activation may be a potential molecular lesion in human BAVM pathogenesis.

In conclusion, this study demonstrates increased activation of endothelial Notch signaling, which we have shown causes BAVM-like abnormalities in mice, in human BAVMs. It suggests that activation of Notch signaling is an important molecular candidate in BAVM pathogenesis, and further validates that our animal model provides a platform to study $\mathrm{BAVM}$ progression and regression. These findings open a new area of research to advance the knowledge and treatment of this devastating disease.

Supplementary Information accompanies the paper on the Laboratory Investigation website (http://www.laboratoryinvestigation.org) 


\section{ACKNOWLEDGEMENTS}

We thank Michael Lawton, MD for neurosurgical perspective, Nathanael Hevelone for statistical advice, members of our laboratory for helpful discussions, and Natasha Cuk for technical support in the early phase of the project. This work was supported by the Pacific Vascular Research Foundation, the Mildred V Strouss Trust, and the Frank A Campini Foundation, to R.A.W. and American Heart Association Predoctoral Fellowship to P.A.M.

1. Friedlander RM. Clinical practice. Arteriovenous malformations of the brain. N Engl J Med 2007;356:2704-2712.

2. Meyer-Heim AD, Boltshauser E. Spontaneous intracranial haemorrhage in children: aetiology, presentation and outcome. Brain Dev 2003;25:416-421.

3. Stapf C, Mohr JP, Choi JH, et al. Invasive treatment of unruptured brain arteriovenous malformations is experimental therapy. Curr Opin Neurol 2006;19:63-68.

4. Thoma R. Untersuchungen über die Histogenese und Histomechanik des Gefässsytems. Ferdinand Enke: Stuttgart, 1893.

5. Wang HU, Chen ZF, Anderson DJ. Molecular distinction and angiogenic interaction between embryonic arteries and veins revealed by ephrinB2 and its receptor Eph-B4. Cell 1998;93:741-753.

6. Gridley T. Notch signaling in vascular development and physiology. Development 2007;134:2709-2718.

7. Lawson ND, Scheer N, Pham VN, et al. Notch signaling is required for arterial-venous differentiation during embryonic vascular development. Development 2001;128:3675-3683.

8. $\mathrm{Kim} \mathrm{YH}, \mathrm{Hu} \mathrm{H}$, Guevara-Gallardo $\mathrm{S}$, et al. Artery and vein size is balanced by Notch and ephrin B2/EphB4 during angiogenesis. Development 2008;135:3755-3764.

9. Hofmann JJ, Iruela-Arispe ML. Notch signaling in blood vessels: who is talking to whom about what? Circ Res 2007:100:1556-1568.

10. Bray SJ. Notch signalling: a simple pathway becomes complex. Nat Rev Mol Cell Biol 2006;7:678-689.

11. Kopan $\mathrm{R}$, Schroeter EH, Weintraub $\mathrm{H}$, et al. Signal transduction by activated mNotch: importance of proteolytic processing and its regulation by the extracellular domain. Proc Natl Acad Sci USA 1996;93:1683-1688.

12. Carlson TR, Yan Y, Wu X, et al. Endothelial expression of constitutively active Notch4 elicits reversible arteriovenous malformations in adult mice. Proc Natl Acad Sci USA 2005;102:9884-9889.

13. Murphy PA, Lam MT, Wu X, et al. Endothelial Notch4 signaling induces hallmarks of brain arteriovenous malformations in mice. Proc Natl Acad Sci USA 2008;105:10901-10906.

14. Roca C, Adams RH. Regulation of vascular morphogenesis by Notch signaling. Genes Dev 2007;21:2511-2524.

15. Garg V, Muth AN, Ransom JF, et al. Mutations in NOTCH1 cause aortic valve disease. Nature 2005;437:270-274.

16. Jarriault S, Brou C, Logeat F, et al. Signalling downstream of activated mammalian Notch. Nature 1995;377:355-358.

17. Shawber CJ, Das I, Francisco E, et al. Notch signaling in primary endothelial cells. Ann N Y Acad Sci 2003;995:162-170.

18. Gale NW, Dominguez MG, Noguera I, et al. Haploinsufficiency of deltalike 4 ligand results in embryonic lethality due to major defects in arterial and vascular development. Proc Natl Acad Sci USA 2004;101:15949-15954.

19. Dou GR, Wang YC, Hu XB, et al. RBP-J, the transcription factor downstream of Notch receptors, is essential for the maintenance of vascular homeostasis in adult mice. FASEB J 2008;22:1606-1617.

20. Lee HY, Wroblewski E, Philips GT, et al. Multiple requirements for Hes 1 during early eye formation. Dev Biol 2005;284:464-478.

21. Blanpain C, Lowry WE, Pasolli HA, et al. Canonical notch signaling functions as a commitment switch in the epidermal lineage. Genes Dev 2006;20:3022-3035.

22. Ridgway J, Zhang G, Wu Y, et al. Inhibition of Dll4 signalling inhibits tumour growth by deregulating angiogenesis. Nature 2006;444: 1083-1087.

23. Fre $S$, Huyghe $M$, Mourikis $P$, et al. Notch signals control the fate of immature progenitor cells in the intestine. Nature 2005;435: 964-968.

24. Riccio O, van Gijn ME, Bezdek AC, et al. Loss of intestinal crypt progenitor cells owing to inactivation of both Notch1 and Notch2 is accompanied by derepression of CDK inhibitors p27Kip1 and p57Kip2. EMBO Rep 2008;9:377-383.

25. Jensen J, Pedersen EE, Galante $P$, et al. Control of endodermal endocrine development by Hes-1. Nat Genet 2000;24:36-44.

26. van Es JH, van Gijn ME, Riccio O, et al. Notch/gamma-secretase inhibition turns proliferative cells in intestinal crypts and adenomas into goblet cells. Nature 2005;435:959-963.

27. Hirata $\mathrm{H}$, Yoshiura $\mathrm{S}$, Ohtsuka $\mathrm{T}$, et al. Oscillatory expression of the bHLH factor Hes 1 regulated by a negative feedback loop. Science 2002;298:840-843.

28. Geffers I, Serth K, Chapman G, et al. Divergent functions and distinct localization of the Notch ligands DLL1 and DLL3 in vivo. J Cell Biol 2007; 178:465-476.

29. Lin MH, Kopan R. Long-range, nonautonomous effects of activated Notch1 on tissue homeostasis in the nail. Dev Biol 2003;263:343-359.

30. Niranjan T, Bielesz B, Gruenwald A, et al. The Notch pathway in podocytes plays a role in the development of glomerular disease. Nat Med 2008;14:290-298.

31. Takeshita K, Satoh M, Li M, et al. Critical role of endothelial Notch1 signaling in postnatal angiogenesis. Circ Res 2007;100:70-78.

32. Tetzlaff MT, Yu W, Li M, et al. Defective cardiovascular development and elevated cyclin E and Notch proteins in mice lacking the Fbw7 F-box protein. Proc Natl Acad Sci USA 2004;101:3338-3345.

33. Yaron O, Farhy $C$, Marquardt $T$, et al. Notch1 functions to suppress cone-photoreceptor fate specification in the developing mouse retina. Development 2006;133:1367-1378.

34. McKellar SH, Tester DJ, Yagubyan M, Majumdar R, Ackerman MJ, Sundt 3rd TM. Novel NOTCH1 mutations in patients with bicuspid aortic valve disease and thoracic aortic aneurysms. J Thorac Cardiovasc Surg 2007;134:290-296.

35. Limbourg A, Ploom M, Elligsen D, et al. Notch ligand Delta-like 1 is essential for postnatal arteriogenesis. Circ Res 2007;100:363-371.

36. Hellstrom M, Phng LK, Hofmann JJ, et al. Dll4 signalling through Notch1 regulates formation of tip cells during angiogenesis. Nature 2007:445:776-780.

37. Venkatesh DA, Park KS, Harrington A, et al. Cardiovascular and hematopoietic defects associated with Notch1 activation in embryonic Tie2-expressing populations. Circ Res 2008;103:423-431.

38. Chen BP, Li YS, Zhao Y, et al. DNA microarray analysis of gene expression in endothelial cells in response to 24 -h shear stress. Physiol Genomics 2001;7:55-63.

39. Dai G, Kaazempur-Mofrad MR, Natarajan S, et al. Distinct endothelial phenotypes evoked by arterial waveforms derived from atherosclerosis-susceptible and -resistant regions of human vasculature. Proc Natl Acad Sci USA 2004;101:14871-14876.

40. Krebs LT, Shutter JR, Tanigaki K, et al. Haploinsufficient lethality and formation of arteriovenous malformations in Notch pathway mutants. Genes Dev 2004;18:2469-2473.

41. Duarte A, Hirashima M, Benedito R, et al. Dosage-sensitive requirement for mouse DIl4 in artery development. Genes Dev 2004; 18:2474-2478.

42. Oda T, Elkahloun AG, Pike BL, et al. Mutations in the human Jagged1 gene are responsible for Alagille syndrome. Nat Genet 1997;16:235-242.

43. Ferrara N, Carver-Moore $\mathrm{K}$, Chen $\mathrm{H}$, et al. Heterozygous embryonic lethality induced by targeted inactivation of the VEGF gene. Nature 1996;380:439-442.

44. Carmeliet $P$, Ferreira V, Breier $G$, et al. Abnormal blood vessel development and lethality in embryos lacking a single VEGF allele. Nature 1996;380:435-439.

45. Raya A, Kawakami Y, Rodriguez-Esteban C, et al. Notch activity acts as a sensor for extracellular calcium during vertebrate left-right determination. Nature 2004;427:121-128.

46. Guo M, Jan LY, Jan YN. Control of daughter cell fates during asymmetric division: interaction of Numb and Notch. Neuron 1996;17:27-41.

47. Begbie ME, Wallace GM, Shovlin CL. Hereditary haemorrhagic telangiectasia (Osler-Weber-Rendu syndrome): a view from the 21st century. Postgrad Med J 2003;79:18-24.

48. Abdalla SA, Letarte M. Hereditary haemorrhagic telangiectasia: current views on genetics and mechanisms of disease. J Med Genet 2006;43:97-110.

49. Matsubara S, Mandzia JL, ter Brugge $\mathrm{K}$, et al. Angiographic and clinical characteristics of patients with cerebral arteriovenous malformations 
associated with hereditary hemorrhagic telangiectasia. AJNR Am J Neuroradiol 2000;21:1016-1020.

50. Bourdeau A, Faughnan ME, McDonald ML, et al. Potential role of modifier genes influencing transforming growth factor-beta1 levels in the development of vascular defects in endoglin heterozygous mice with hereditary hemorrhagic telangiectasia. Am J Pathol 2001;158:2011-2020.

51. Arthur HM, Ure J, Smith AJ, et al. Endoglin, an ancillary TGFbeta receptor, is required for extraembryonic angiogenesis and plays a key role in heart development. Dev Biol 2000;217: 42-53.

52. Torsney E, Charlton R, Diamond AG, et al. Mouse model for hereditary hemorrhagic telangiectasia has a generalized vascular abnormality. Circulation 2003;107:1653-1657.

53. Li DY, Sorensen LK, Brooke BS, et al. Defective angiogenesis in mice lacking endoglin. Science 1999;284:1534-1537.

54. Bourdeau A, Faughnan ME, Letarte M. Endoglin-deficient mice, a unique model to study hereditary hemorrhagic telangiectasia. Trends Cardiovasc Med 2000;10:279-285.

55. Srinivasan $\mathrm{S}$, Hanes MA, Dickens $\mathrm{T}$, et al. A mouse model for hereditary hemorrhagic telangiectasia (HHT) type 2. Hum Mol Genet 2003;12:473-482.

56. Hashimoto $\mathrm{T}$, Lawton MT, Wen G, et al. Gene microarray analysis of human brain arteriovenous malformations. Neurosurgery 2004;54: 410-423; discussion 423-425.

57. Yang GY, Xu B, Hashimoto $T$, et al. Induction of focal angiogenesis through adenoviral vector mediated vascular endothelial cell growth factor gene transfer in the mature mouse brain. Angiogenesis 2003;6:151-158.

58. Xu B, Wu YQ, Huey $M$, et al. Vascular endothelial growth factor induces abnormal microvasculature in the endoglin heterozygous mouse brain. J Cereb Blood Flow Metab 2004:24:237-244.

59. Zhu Y, Lee $C$, Shen F, et al. Angiopoietin-2 facilitates vascular endothelial growth factor-induced angiogenesis in the mature mouse brain. Stroke 2005;36:1533-1537.

60. Zhu Y, Shwe Y, Du R, et al. Effects of angiopoietin-1 on vascular endothelial growth factor-induced angiogenesis in the mouse brain. Acta Neurochir Suppl 2006;96:438-443.

61. Hao Q, Su H, Marchuk DA, et al. Increased tissue perfusion promotes capillary dysplasia in the ALK1-deficient mouse brain following VEGF stimulation. Am J Physiol Heart Circ Physiol 2008;295:H2250-H2256.

62. Hashimoto T, Lam T, Boudreau NJ, et al. Abnormal balance in the angiopoietin-tie2 system in human brain arteriovenous malformations. Circ Res 2001;89:111-113.

63. Limaye $N$, Wouters $V$, Uebelhoer $M$, et al. Somatic mutations in angiopoietin receptor gene TEK cause solitary and multiple sporadic venous malformations. Nat Genet 2009;41:118-124.

64. McCarty JH, Lacy-Hulbert A, Charest A, et al. Selective ablation of alphav integrins in the central nervous system leads to cerebral hemorrhage, seizures, axonal degeneration and premature death. Development 2005;132:165-176.

65. ApSimon HT, Reef H, Phadke RV, et al. A population-based study of brain arteriovenous malformation: long-term treatment outcomes. Stroke 2002;33:2794-2800. 